\title{
Bioinformatics, Healthcare Informatics and Analytics: An Imperative for Improved Healthcare System
}

\author{
Jelili Oyelade \\ Department of \\ Computer and \\ Information Sciences \\ Covenant University \\ PMB 1023, Ota, Ogun \\ State, Nigeria
}

\author{
Jumoke Soyemi \\ Department of \\ Computer Science \\ Federal Polytechnic, \\ Ilaro, Ogun State, \\ Nigeria
}

\author{
Itunuoluwa Isewon \\ Department of \\ Computer and \\ Information Sciences \\ Covenant University
}

\author{
Olawole Obembe \\ Department of \\ Biological Sciences \\ Covenant University \\ PMB 1023, Ota, Ogun \\ State, Nigeria
}

\begin{abstract}
Healthcare Informatics focuses on health data, information and knowledge, including their collection, processing, analysis and use. Bioinformatics employ computational tools and techniques to study and analyse large biological databases and to absolutely understand disease and grasp the genetics and proteomics by relating them with healthcare data. The focus is on processing genomic and proteomics data for basic research in biology, but also medicine, drug discovery, and related areas. Analytics in healthcare came as a result of large healthcare data that are being gathered electronically. Data analytics is proficient in terms of healthcare improvement, reduction in cost and safety of lives. Applications of data analytics in healthcare is as a result of the eruption in data to mine understandings so as to make informed decisions. This paper reviews bioinformatics, Healthcare Informatics and Analytics as an imperative for an improved Healthcare System. It looks at the benefits, the contribution of each of them to improving healthcare system, the overlap among bioinformatics, healthcare Informatics and analytics and finally the future prospects of healthcare informatics and analytics.
\end{abstract}

\section{Keywords}

Healthcare Informatics, Bioinformatics, Analytics, Healthcare Syatem

\section{INTRODUCTION}

Healthcare is an important sector of any economy; as such for a society to realize its dream and vision of sustainable growth, it is important that physical and mental health of its individuals is ensured. The development of a nation is therefore dependent on the state of health of the citizens, hence the popular saying "health is wealth". Therefore, any technology or system that is tailored towards improving the healthcare system is most likely to enjoy favorable public perception. Ever the time the Human Genome Project was completed in 2003, opportunities came to be able to acquire such data, analyze, and present the genomic data in a faster, cheaper, and reliable way. This dramatic technological advance has affected healthcare in term of new prevention development, better diagnosis, and improved treatment for regular healthcare. These large volumes of various data from bioinformatics and healthcare informatics domains coupled with analytics are estimated to deliver in the near future preventive, predictive and personalized healthcare aids. This is expected to contribute to national growth and development.

\section{HISTORICAL DEVELOPMENT OF BIOINFORMATICS}

The history of bioinformatics dates back to 1981 when 579 human genes were mapped by in situ hybridization. There was huge movement in bioinformatics when Marvin Carruthers and Leory Hood in 1981, invented an automated DNA sequencing method. The international organization of scientists involved in Human Genome Project, Human Genome Organization (HUGO) was established in 1988 and published the first complete genome map in 1989.

The Project began in 1990 and in 1991, a total of 1879 human genes were mapped. Also in 1993, a human genome physical map was produced in France by Genethon, a research centre that specialises in human genome [1]. The first phase of the project on Human Genome was concluded after three years when Genethon published the final report on the Human Genetic Map [2]

Having completed the Human Genome project in the year 2003, biologists, computer scientists and statisticians came together to form the bioinformatics discipline. The Bioinformatics arose as a need to analyze the genome data and get a grounded understand of those genes that are related to some diseases as well as uncover the complexity of their biological activities [3]. Bioinformatics is therefore an interdisciplinary area that involves the management, analysis and interpretation of information from biological sequences and structures.

The demand to produce enormous databases, for instance EMBL, GenBank and DNA further enhanced Bioinformatics, to store and match the DNA sequence data emanating and produced by the human genome and other projects on genome sequencing. Currently, bioinformatics encompasses functional details of protein/gene, structural analysis of protein, preclinical and clinical trials data from patients and the metabolic pathways of various species [3].

\subsection{The rationale for Bioinformatics}

Bioinformatics provided opportunity for the researchers to store data, such as DNA sequencing, its analysis and interpretation, on computers in the form of databases. Biological data in its raw form without compilation cannot be used even by professional researchers. Bioinformatics has thus enabled scientists to develop biological tools to source information from these databases that can be used for the purpose of research. Bioinformatics tools are vital to determine protein sequences, structures and functions. The 
complete knowledge of these three gives a better understanding of the biology of an organism.

Raw biological data can be easily stored as databases in the computers but extracting the required information from the quantum of data is the actual challenge for researchers. For this reason, bioinformatics tools perform a vital role in extracting and analyzing information from the databases. The bioinformatics tools are designed, such that it is easy to use by the biologists.

\subsection{Bioinformatics and its Contribution to}

Bioinformatics originally came as a result of the need to give direction to the enormous data generated through molecular biology technology. The technology produced DNA sequencing in a large scale and also tools to establish positions of protein coding areas in DNA. In addition, the creation of sequence repositories was done at the same time

Multiple gene expression measurement is an additional technology, which is already providing wealth of new data. The concept is to uncover DNA chip containing DNA nucleotide fragments in thousands, with individual identifying a gene exclusively to coding DNA obtained from a sample of interest. In order to locate subsets of genes, multiple gene expression methods are often used. This gives room to be able to differentiate between more than two biological situations also known as supervised classification. Unsupervised classification locates clusters in the gene sample space resulting in the classification of samples and genes. It is the analysis of gene expression data that brought about advances in computational algorithms from computational methods with their foundation in Computer science and statistics [4].

Remarkable feats have also been accomplished in data mining methods for genomics and proteomics. The successes have enhanced and improved the insight into genes related with some diseases and the discovery of novel drugs that are very effective. Also many national, international committees and groups are encouraging insertion of genetic information in electronic health records (EHRs). The groups' attempt is bound to have a far-reaching influence on personalized medicine. Genetic research advancement has sharpened the approach for the cutting-edge vision of personalized medicine, that determines the correct medicine for the patient using the patient's genetic composition [5].

\section{HEALTHCARE INFORMATICS}

Healthcare informatics deals with the best use of information, through the help of technology, in improving healthcare, public health, and biomedical research [6]. It is more about information than the technology. [7]. Informatics is a blend of life-health sciences and computer and informatics sciences to make people better [8].

Healthcare informatics, in the simplest definition, is the division of healthcare that is involved in providing the right and useful information about a patient's state of health to the right person, the healthcare professionals, at the right time it is required, thereby guiding them in making and taking informed decisions about their patient's treatment. There is a synergy and exchange of information in Healthcare informatics between patients, healthcare professionals, healthcare planners, Internet Technology providers and management of healthcare facilities.
According to the US Department of Health [9], "healthcare informatics is defined as the knowledge, skills and tools which enable information to be collected, managed, used and shared to support the delivery of healthcare and promote health." The evolution of Healthcare informatics came from the transformation of traditional healthcare delivery system to the information age healthcare delivery system. The name healthcare informatics originated from the coalition of similar terms (though they are sometimes used interchangeably, but there are lots of variances between them), these are: electronic health, popularly known as eHealth, Information management and technology also known as IM\&T, Medical informatics and Telehealth.

Healthcare Informatics combines information and understanding from medical areas (pre-clinical, clinical and post-clinical), healthcare administration and management and information technology. Health Informatics experts understand information technology, application of technology to solve real life challenges and managing multifaceted processes of executing novel answers into organizations. Besides these common capabilities, an expert in Health Informatics is also conversant with the industry, both from the standpoint of offering medical services, and from the standpoint of managing, challenging and demanding medical organizations and procedures.

Medical Informatics makes available the essential tools to relate data and knowledge in the process of decision making. Information management and technology ensures collection and information management from various sources, including the processing and delivery of the same. The objective is to assist in health care, increase the performance within organization, resolve business issues as well as assist service and business plans.

eHealth is a new term often used in European countries that implements electronic and digital technologies for healthcare practices, processes and communications.

Telehealth combines technology with health services that gives opportunity to people with medical conditions to access healthcare within the comfort of their home. A home Pod device installed in a patient's home is an example of telehealth that can be used to monitor the patient's health condition, the information that can then be communicated securely over the server to a remote clinician.

\subsection{Why Healthcare Informatics}

The healthcare sector is growing rapidly as one of the most significant sectors of contemporary society. In 2009, the Obama administration invested $\$ 50$ billion to encourage health information technology and electronic prescription. The sector accounts for a significant share of public expenditure and this is motivated by persistent demands to innovate.

The detonation of biological and medical information has led to major increases in the information management which is vital for health professionals. Health informatics professionals are specialists in managing this information in support of health professionals.

Information is the bedrock of the healthcare industry. Information about patient conditions is essential and must be up-to-date, consistent and accurate for providing medical services. Also too, for proper coordination of the many 
organizations and individuals involved in each medical service, information is required. Furthermore, information is necessary to administer the complex economic dealings entailed in the industry.

There have been considerable challenges in implementing information technology for the promotion of efficiency and effective qualitative medical services due to the complexity, intensiveness and the volume of healthcare information. To this end, innovation in the healthcare industry is driven and dependent on highly skilled health informatics personnel.

The emphasis in healthcare presently is to minimize the volume of waste in healthcare by carrying out new systems of health Information Technology (healthcare informatics) reducing inefficiency, redundancy and administrative cost which therefore, promotes timeliness, transparency and improved outcome in healthcare delivery.

\subsection{Healthcare Informatics and its Contribution to Healthcare System}

Healthcare informatics is a branch of learning at the interphase of healthcare and information science. It involves the resources, devices and methods necessary to enhance the process of acquiring, storing, recovering and usage of information in health and biomedicine. The tools used in Health Informatics include computers, information and communication systems, formal medical vocabularies and clinical procedures.

Health Informatics permits an organized way of storing and retrieving information which is more precise than the recall of a patient for details of the present medications and allergies. This is the most important safety issue for patient. Inaccurate or inadequate information from a baffled patient can lead to an adverse drug effect. Therefore, providing accurate information when needed is very essential.

Health Informatics facilitates joined-up care, where different departments, such as laboratory, surgery, radiology, account or administrative section, are connected together thus reducing the duplication of efforts and speeds up processes. This time-saving feature is especially crucial with the growing needs in healthcare.

It also constitutes a tool for decision-making. Computerized guidelines offer the advantages of helping patients and clinicians make better decisions. Due to this, high quality prescription and treatment can be sustained. Moreover, doctors and other health personnel can have more quality time with the patients because of less time on administrative work.

Health Informatics eradicates the need to keep logging the same date again and again. It minimizes wastage. Also health Informatics ensures improvements that facilitate efficient delivery of quality healthcare. It is therefore a field that combines information science, information system, technology and healthcare. Health informaticians' expertise is engaged for resource management, medical care standardization, research coordination and efficient delivery of healthcare.

\section{HEALTHCARE ANALYTICS}

According to IBM institute for Business value [10], "Analytics is the systematic use of data and related business insights developed through applied analytical disciplines such as statistical, contextual, quantitative, predictive, cognitive, and other including emerging models to drive fact-based decision making for planning, management, measurement and learning. Analytics may be descriptive, predictive or prescriptive."

Healthcare in the United States has continued to advance within three phases of data management, talking about data collection, data sharing and data analytics. Data collection and data sharing phases are what gave rise to the urgent distribution of electronic health records as well as health information exchanges. A key potential of analytics rests in its capability to change healthcare into a truly data-driven system [11].

In the years ahead, a dramatic and sporadic growth is expected in Health data [12]. Likewise, healthcare reimbursement models are transforming; significantly pay as you use are emerging as vital new factors in today's healthcare industry. Healthcare organizations should not be primarily motivated by profits, it is vital that they acquire the accessible tools, facilities, and procedures to control big data effectually otherwise risk losing huge sum of money in revenue and profits [13].

Generally, the healthcare system already produced huge dataset, propelled by record keeping, compliance and regulatory obligations, and patient care [14]. The convention is to have data stored in paper form but the present drift is swift digitization of all these huge dataset. This trend is motivated by the binding and the prospective to advance as well as improve the standard of healthcare delivery at the same time ensuring cost reduction. These huge quantities of data also called big data, guarantees the support of a large number of medical and healthcare tasks ranging from clinical decision support, disease surveillance, and population health management $[15,16,17]$.

In 2011, data from U.S. healthcare system attained 150 exabytes, it is believed that with this proportion, healthcare in the U.S. will rapidly approach zettabyte $\left(10^{21}\right.$ gigabytes $)$ scale and sooner yottabyte ( $10^{24}$ gigabytes) [12].

\subsection{Why Healthcare Analytics}

Analytics in healthcare came as a result of large volume of health data which is being collected. These data includes electronic patient records, key national initiatives and databases, which link patient data throughout hospitals, regions and so on.

Another need for healthcare analytics is the being able to obtain various data from patient, patient being able to access their own data and additional health information (Personal health records) and finally the being able to work across these multiple data to identify patient and population health needs, optimizing and streamlining the healthcare system and properly evaluating information system [18].

The unnerving challenges confronting the healthcare industry presently necessitate persuasive advices to enlarge the analytics roles. There are proofs that healthcare is progressively confronted by entrenched ineptitudes, and wasting over US\$2 trillion yearly [19]. The lack of efficiency is due to inefficient gathering, sharing and use of information [20].

In the United States, 96 people out of 100,000 die annually from health situations amenable to healthcare, the outcome of clinical remains suboptimal in this instance. Reports from 
hospitals in Australia, Canada, Denmark, France, New Zealand, Spain, the United Kingdom and the United States, have it that there are high levels of preventable errors. Error rates ranged from 2.9 to 45.8 percent for hospitalized patients, of which 7.6 to 51.2 percent were preventable [21]

Apart from systemic problems, other factors contribute to the vast difficulty the healthcare industry is experiencing. There are high hopes from citizens of their healthcare providers, since they have more access to information than before, resulting in demand of increasing accountability from their doctors, nurses and health policies. In fact, from the consumer point of view, health plans ranked last among 14 sectors in a customer experience survey, trailing even television and Internet service providers, and well behind other insurance providers [21]. With the government's regulatory presence on the increase, additional focus is given to accountability, governance and oversight on the sector [22].

Exploiting analytics for better understanding will help in achieving the best output, such as new therapies and technologies. Insight through Information also could assist educated learned and informed consumers become more accountable for their own health. Analytics will therefore improve efficiency, aid discovery and exploration, improve service delivery and operations and serve as a means to measure and evaluate critical organizational data. In addition analytics can also increase healthcare access, performance align with pay and healthcare costs stability.

\subsection{Healthcare Analytics and its Contribution to Healthcare System}

According to Ravi [23], what is driving analytics adoption in healthcare is that there are huge amount of data but little information and knowledge. There are a number of benefits accrued to healthcare system implementing analytics tools and processes. These include;

- solution of healthcare management which incorporate health and wellness

- improved real-time decisions about treatment and support, consumer commitment

- better revenue management with insights into local markets

- health management tools integrated with clinical analytics and communication technologies

- Pay for Quality reimbursement change predictability, workflows and analytics

- Mining for unknown variables that influence quality such as "hidden" readmission factors

- Evidence-Based Medicine, which standardizes clinical processes, protocols, and guidelines, Claims data analysis for fraud" and so on.

According to Wullianallur and Viju [24], analytics has potential to uncover relationships and identify trends and patterns within data. Data analytics is proficient in terms of healthcare improvement, reduction in cost and safety of lives [25]. Application of data analytics in healthcare is as a result of the eruption in data to mine understandings thus making knowledgeable decisions. [26, 27]. A research category in this regard is referred to as big data analytics in healthcare $[28,22$, 29].

\section{THE OVERLAP AMONG BIOINFORMATICS, HEALTHCARE INFORMATICS AND ANALYTICS}

Bioinformatics, Health informatics and Analytics involve the development of storage, analytic and interpretive methods to optimize the revolution of progressively huge biomedical data into hands-on, prognostic, preventive and participatory health. The use of informatics by the medical group is growing as computational proficiency and resources continue to improve [30]. A remarkable revolution in this section is the EHRs development that made available data rich warehouse and the prospect to uncover genotype and phenotype relationship, thus supporting healthcare in using genomic result for improved patient care.

Healthcare Informatics focuses on health data, information and knowledge, including their collection, processing, analysis and use. The focus is on data and its use in decisionmaking. Computer science and information technology only provide tools in doing so.

Bioinformatics thus employ computational tools and techniques to study and analyze large biological databases and to absolutely understand disease and grasp the genetics and proteomics by relating them with healthcare data. The discipline focuses on processing genomic and proteomics data for basic research in biology, but also medicine, drug discovery, and related areas.

The knowledge of the genetic mechanism of a disease makes it simple for the physician to diagnose more correctly. One of the upcoming prospects is possibility of using genes instead of medicines to cure specific disease by varying diseased protein expression. This opportunity is enhanced by gene therapy techniques. Although, the technique is still in the trial stages but there are positive likelihoods that in the future, the technique will be recognized.

Biological and bioinformatics dataset are growing exponentially and thus present opportunities as well as challenges to researchers to contribute and help understand the genetic basis and phenotypes. The breakthrough in microarray technology created the possibility to concurrently oversee several thousands of genes expression. It is highly important for researchers to gain access to the healthcare data so as to get better insight into the genetics and proteomics of the diseased tissue. The technology may possibly lead to a major breakthrough in personalised medicine with storage provision for healthcare and genetic data in electronic health records.

Biology and medicine have therefore evolved from benchbased to computer-based science. Experiments are replaced with models. To further advance research on biomedical demands an intersection of genomic and healthcare research. Tremendous improvement in collaborative research as well as the development of advanced and innovative tools and technologies to boost patient care is achieved by the integration of information at the molecular, cellular, tissue and personal level [5]. 


\section{FUTURE PROSPECTS OF HEALTHCARE INFORMATICS AND ANALYTICS}

The genomic technologies have the capacity to advance diagnosis, treatment of diseases especially inherited diseases and intricate diseases such as cancer and accelerate the shift in the direction of personalized and predictive medicine. The huge genomic data and computational challenges due to high throughput as well as falls in the next generation sequencing has resulted in movement away from this research to clinical implementation with careful integration with healthcare information. The primary purpose of integrating biology and healthcare information is to give rooms for exchanges in technology, data, and knowledge. This will in turn provide a database of diseases, their causes, the trigger patterns and possible treatment and cure which will speed up healthcare access and thus provide a reliable and efficient healthcare system. The introduction of analytics emerged to save the day in the healthcare industry in terms of huge amount of data collected electronically across heterogeneous platforms. Analytics can thus help to generate insights that lower costs, reduce inefficiencies, improve outcomes, identify at-risk populations; predict individuals' future healthcare needs, empower patient and support physicians' diagnoses.

\section{CONCLUSION}

The revolution presented by genomic technology when incorporated with health informatics data will go a long way in improving the present healthcare system while analytics will enable more efficient use of resources by ensuring that those who need care the most receive it. It is therefore imperative that researchers pay more attention to the integration of the three hot fields (Bioinformatics, Health informatics and analytics) to make the most of it for innovations, creativities and enhanced health care of the people.

\section{REFERENCES}

[1] Hogeweg, P. 2011. "The Root of Bioinformatics in Theoretical Biology". Plos Computationa Biology, 7(3).

[2] Fleischmann R.D., Adams M.D., White O., Clayton R.A., Kirkness E.F., Kerlavage A.R., Bult C.J., Tomb J.F., Dougherty B.A., Merrick J.M. 1995. "Wholegenome random sequencing and assembly of Haemophilus influenza”. Science 269 (5223): 496-512.

[3] Muller, U. R., and Nicolau D. V. 2004. "Microarray Technology and Its Applications". Berlin: Springer. 361374

[4] Andrzej Polanski and Marek Kimmel 2007. Bioinformatics. New York: Springer Verlag Berlin Heidelberg.

[5] Prerna S., and Kimberly T., 2009. "Translational Bioinformatics and Healthcare Informatics: Computational and Ethical Challenges". Online Research Journal Perspectives in Health Information Management, 6.

[6] Hersh W. 2009. "A stimulus to define informatics and health information technology". BMC Med Inform Decision Making 9:24

[7] In Y. C., Tae-Min K., Myung S. K., Seong K. M. and Yeun-Jun C. 2013. Perspectives on Clinical
Informatics: Integrating Large-Scale Clinical, Genomic, and Health Information for Clinical Care. Genomics and Informatics. Published online by Korea Genome Organization.

[8] Friedman, C. 2009. "A fundamental theorem of biomedical informatics". Journal of the American Medical Informatics Association, 16: 169-170.

[9] US Department of Health. 2002. Making information count: a human resources strategy for health informatics professionals. USA: Department.

[10] IBM Institute for Business Value 2012. The Value of Analytics in healthcare. IBM Global Business Services, USA.

[11] Dale, S., David, A. S. and Denis, P. 2013. The Healthcare Analytics Adoption Model: A Framework and Roadmap. White paper by Health Catalyst.

[12] IHIT, 2013. Transforming healthcare through Big data strategies for leveraging health care industry. The Institute for Health Technology Transformation.

[13] LaValle, S., Lesser E., Shockley R., Hopkins M.S., Kruschwitz N. 2011. "Big data, analytics and the path from insights to value". MIT Sloan Manag Rev,52:20-32.

[14] Raghupath, W. 2012. Data Mining in Health Care. In Healthcare Informatics: Improving Efficiency and Productivity, pp. 211-223.

[15] Burghard C. 2012. Accountable Care driven Big data and analytics. White Paper by IDE Health insights.

[16] Dembosky A. 2012. Data prescription for better healthcare. Financial Times, pp. 19.

[17] Fernandes L., O'Connor M., Weaver V. 2012. "Big data, bigger outcomes: Healthcare is embracing the big data movement, hoping to revolutionize HIM by distilling vast collection of data for specific analysis". J AHIMA 83(10):38-43.

[18] André W. K. 2008. Forecasting in Health Care: Integrating Analytics with Electronic Health Records SAS Institute Inc.

[19] Korsten, P. and Christian S. 2010. The world's 4 trillion dollar challenge. Using a system-of-systems approach to build a smarter planet. IBM Global Business Services.

[20] The Commonwealth Fund, 2011. Commonwealth Fund National Scorecard on U.S. Health System Performance. Source: Commonwealth Fund National Scorecard on U.S. Health System Performance, 2011.

[21] Adams, J. R., Bakalar, M. D., Michael B., Karen K., Edgar L. M. and Neil S. 2008. "Healthcare 2015 and care delivery: Delivery models refined, competencies defined." IBM Institute for Business Value.

[22] IBM 2012. IBM big data platform for healthcare. Solution Brief.

[23] Ravi, K. 2013. Informatics or Analytics? Understanding Healthcare Provider Use cases. Retrieved September 10, 2014 from: http://practicalanalytics.wordpress.com/2013/07/15/infor $\mathrm{m}$ atics-or-analytics-understanding-healthcare-provideruse-cases/ 
[24] Wullianallur R. and Viju R. 2014. "Big data analytics in healthcare: promise and potential. Health Information" Science and Systems, 2:3

[25] IKANOW 2012. Data analytics for healthcare: creating understanding from Big data. Retrieved August 14, 2014 from: http://info.ikanow/portals/163225/docs/dataanalytics-for-healthcare.pdf

[26] Carlton, M. 2012. How big data analytics reduced medical re-admissions. IBM Corporation.

[27] Knowledgent 2012. Big data and healthcare payers. White Paper by Knowledgent Innovation through Information. Knowledgent White Paper Series.
[28] Anil, J. 2009. Unlocking the power of big data to improve healthcare for everyone. Explorys.

[29] Intel 2012. Leveraging Big data and analytics in heathcare and life sciences: enabling personalized medicine for high-quality care, better outcomes. Medtech media.

[30] Greenes, R. A. 1994. Strategic planning activities of American Medical Informatics Association. J Am Inform Assoc, 1(3):263-271. 